\section{MS34-P8 Cavity analysis in preparation of} new solid-state phases of small molecules

Radka Zajicova $^{1}$, Jan Čejka ${ }^{1}$, Alexandr Jegorov ${ }^{2}$

1. Department of Solid State Chemistry, University of Chemistry and Technology Prague, Technicka 5, Prague 6, Czech Republic

2. Teva Czech Industries s.r.o. , Research Unit, Branišovská 31,

37005 České Budějovice, Czech Republic

email: zajicovr@vscht.cz

Most solid substances exist in various forms, either polymorph or multi-component phases. The particular forms often differ in structure and physico-chemical properties. Plenty of ways are available to analyse and characterize solid phases. Even more points of view and software methods are used to examine and compare the obtained data sets. Cavity analysis of solid substances is a typical research area for biological macromolecules, zeolites and MOFs. Nevertheless it proves a great instrument in small molecules crystallography. Voids in crystal structures of small molecules are generated by packing of molecules of interest. Frequently, the primary molecules build voids, which can be occupied by solvent or co-former molecule, or just remain empty. The resulting molecular packing depends on crystal growth conditions and stability of the substance. The most important tracked parameters are: size, shape and position of each particular void in unit cell or in neighbour unit cells. This study applies cavity analysis in looking for new solid-state phases when at least one solid form is known. It is based on similarities of shape and size of cavities and shape and size of incorporated molecules (i.e. solvents) defined by different approaches. This work is supported by the Grant Agency of Czech Republic, Grant no. $106 / 14 / 03636 \mathrm{~S}$.

Keywords: multi-component phases, cavity analysis
MS34-P9 Inclusion compounds of a borneol dumb-bell host with methylcyclohexanones and 2-butanols: Structures and resolutions

Eustina Batisai ${ }^{1}$, Luigi R. Nassimbemi ${ }^{1}$, Edwin Weber ${ }^{2}$

1. Department of Chemistry, University of Cape Town, Rondebosch 7700, South Africa

2. Institut für Organische Chemie TU Bergakademie Freiberg Leipziger Strasse 29, D- 09596 Freiberg/Sachs., Germany

\section{email: ebatisai@gmail.com}

Functionality and bulkiness are structural criteria typical of host molecules designed for crystalline inclusion formation. ${ }^{1}$ Molecules meeting these requirements have been developed in a variety of geometric structures ${ }^{2}$ including those resembling the shape of a wheel-and-axle ${ }^{3}$ or a dumb-bell. ${ }^{4}$ In a special kind of purpose-built host structures of the dumb-bell kind, chiral borneol moieties were used as bulky terminal substituent groups, thus providing chirality and hydrogen bonding sites. In this work, the host 2,2'-(benzene-1,4-diyl-diethynylene) diborneol has been employed to resolve racemic methylcyclohexanones and 2-butanols. For 2-methylcyclohexanone, the resultant inclusion compound yielded an enantiomeric excess of $72 \% \quad(S)$ while with 3-methylcyclohexanone the enantiomeric excess was $57 \%(S)$. The host failed to resolve $(R, S)$ - 2-butanol, and the inclusion compounds derived from $(R, S)-,(R)-$ and $(S)-$ 2-butanol are isostructural, being dominated by a stable framework of host $\bullet \bullet$ host hydrogen bonds. The non templating effect of the 2-butanols was explained in terms of the secondary interactions occurring in the structures which were also analysed by the program CrystalExplorer.

1. E. Weber In: Inclusion Compounds; Eds., J. L Atwood, J. E. D. Davies, D. D. MacNicol Oxford University Press: Oxford, 1991, vol. 4, pp. 188-262

2. R. Bishop, Chem. Soc. Rev. 1996, 311

3. L. R. Nassimbeni, H. Su, T-L Curtin, Chem. Commun. 2012, 48, 8526

4. T. Müller, J. Hulliger, W. Seichter, E. Weber, T. Weber, M. Wübbenhorst, Chem. Eur. J., 2000, 6, 54

Keywords: Chiral host, resolution, methylcyclohexanones, 2-butanols 